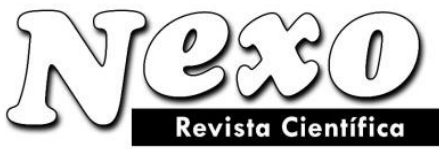

Vol. 33, No. 01, pp. 137-147/Junio 2020

\title{
ASYMMETRIC RECONFIGURATION OF A 3-DOF PARALLEL MANIPULATOR
}

\section{RECONFIGURACIÓN ASIMÉTRICA DE UN MANIPULADOR PARALELO DE 3-GDL}

\author{
A. L. Balmaceda-Santamaría ${ }^{1, *}$, A. E. Chávez-Toruño ${ }^{2}$ \\ ${ }^{1}$ Universidad Nacional de Ingeniería. Facultad de Tecnología de la Industria, Instituto de Estudios \\ Superiores. Managua, Nicaragua. \\ ${ }^{2}$ Universidad Nacional de Ingeniería. Facultad de Ciencias y Sistemas, Departamento de Informática. \\ Managua, Nicaragua. \\ *Albert.Balmaceda@ fti.uni.edu.ni
}

(recibido/received: 29-enero-2020; aceptado/accepted: 23-abril-2020)

\begin{abstract}
In this paper an asymmetric reconfigurable parallel manipulator is presented. Asymmetric configurations are obtained by modifying the angle between each of the kinematic chains. Thanks to the reconfiguration proposal significant improvement of the manipulator performance can be obtained with respect to a Deltatype parallel robot. The computation of the best condition number is obtained, the results show that by using the redundancy, all the performance indices that depend on the Jacobian matrix can be improved as well.
\end{abstract}

Keywords: Parallel manipulator; Performance index; Reconfigurable robot; Screw theory; Workspace.

\section{RESUMEN}

En este trabajo se presenta un manipulador paralelo reconfigurable asimétrico. Al modificar los ángulos de cada una de las cadenas cinemáticas pueden obtenerse configuraciones asimétricas. Gracias a la reconfiguración propuesta pueden generarse mejoras significativas del desempeño del manipulador, con respecto a un robot paralelo tipo Delta. El cálculo del mejor número de condición es obtenido, los resultados muestran que usando la redundancia todos los índices de desempeño que dependen de la matriz Jacobiana pueden ser mejorados también.

Palabras claves: Espacio de trabajo; Índice de desempeño; Manipulador paralelo; Robot reconfigurable; Teoría de tornillo. 
A. L Balmaceda-Santamaría and A. E. Chávez-Toruño.

\section{INTRODUCTION}

The flexible manufacturing system is a highly versatile production system, which can adapt to different requirements of the market. Reconfigurable manufacturing parallel manipulators can quickly adjust their structure according to a specific task, which is desirable to satisfy market needs and suitable for flexible manufacturing systems.

In robotics the reconfiguration concept was presented by (Krefft, et al., 2006) as the change of the characteristics of the robot in operation, distinguished in two types: static and dynamic. The main approaches that have been proposed for reconfiguration of parallel mechanisms as the Gough-Stewart platform are modular and variable geometry designs, see for instance (du Plessis and Snymar, 2006; Kumar, Nagarajan and Srinivasa, 2009; Chen, 2012).

A modular manipulator consists of a set of modules that can be assembled into robots with different attributes. The modularity has been used recursively in parallel manipulators by (Zhiming and Phillip, 1998; Gogu, 2007; Plitea et al., 2013).

The reconfiguration of parallel manipulators using variable geometry consists of changing some dimensions of the robot with the purpose to generate new postures upon the same parallel manipulator. A double planar parallel reconfigurable manipulator presented by (Simaan and Shoham, 2003), the reconfigurable platform based on the Stewart-Gough of (Borràs, et al., 2009), a reconfigurable parallel mechanism with an adjustable base designed by (Zhang and Shi, 2012) and a new family of reconfigurable parallel mechanisms (Ye, et al., 2014), are some which may be mentioned.

Furthermore, many authors (including some aforementioned) have used the redundancy produced sometimes by reconfigurations of the parallel robots for improving characteristics such as: stiffness (Kock and Schumacher, 1998; Bi and Kang, 2010; Moosavian and Xi, 2014), force (Jiang, Li and Wang, 2015a; Li, Zhang and Zhao, 2014), accuracy (Kotlarski, Heimann and Ortmaier, 2010), increased workspace and singularity-free workspace (Kotlarski, Heimann and Ortmaier, 2012; Cha, Lasky and Velinsky, 2007; Xu, et al., 2010), payload-capacity (Chen, 2012; Yue, Tso and Xu, 2001), as well as performance indices as manipulability, condition number, global condition, dexterity and global dexterity (Dan, Rui and Wuyi, 2014; Finistuari and Xi, 2013; Jian, Li and Wang, 2015b; Wu, Wang and Wang, 2008; BalmacedaSantamaría and Chávez-Toruño, 2019).

It is evident that reconfigurable parallel manipulators are a well-documented problem, since the reconfigurability remains today a challenge in the parallel robotic field, mainly in issues of occupy large structural spaces. In the case of the mechanisms aforementioned, they have a high-level mechanical complexity in their structures and hence very expensive to be made.

In this paper an asymmetric reconfiguration of a 3-DOF parallel manipulator, based on the concept of variable geometry is presented. The base robot used for the reconfiguration proposal, named Parallix LKF2040, was designed as a didactic version at National Polytechnic Institute (IPN) in CICATA, Mexico (Gonzalez-Hernandez and Castillo-Castaneda, 2013). The reconfiguration strategy is highly versatile, very simple and it is capable to return to the original configuration and mechanical characteristics of the Parallix LKF-2040.

The redundancy generated in the manipulator by the reconfiguration proposal, is used to improve the kinematic performance of the robot, by calculating a performance index along prescribed trajectories.

Nexo Revista Científica / Vol. 33, No. 01, pp. 137-147 / Junio 2020 


\section{DESCRIPTION OF THE RECONFIGURABLE ROBOT}

The Parallix LKF-2040 robot is a translational manipulator that was developed at IPN - CICATA. This manipulator is very used for teaching purposes, since that it has an open architecture (Gonzalez-Hernandez and Castillo-Castaneda, 2013).

The Parallix robot comprises 3 stationary motors disposed angularly on the robot base through brackets. The motor axes are coupled to a kinematic chain at the bracket level. This mechanism has a structure of the well-known Delta robot, however the mechanism of Fig. 1 is a 3-RUU robot.

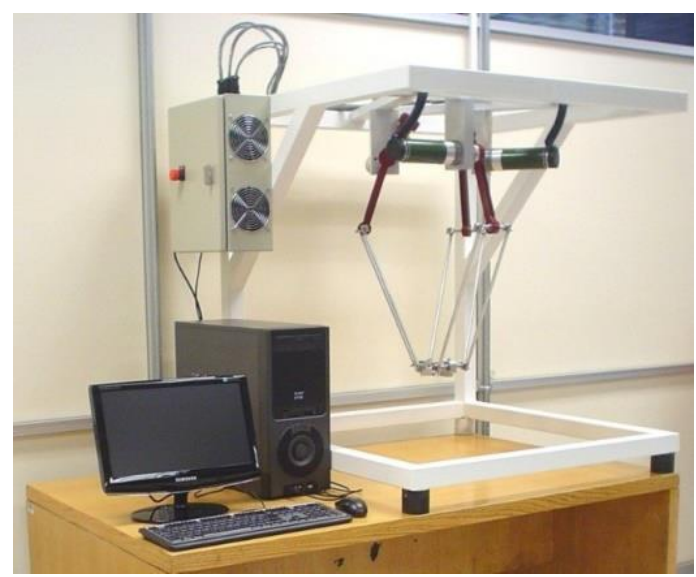

Figure 1. Configuration of the Parallix robot.

\subsection{Asymmetric reconfiguration strategy}

The main variable that significantly influences in the Parallix workspace is the diameter of the fixed platform, since the diameter length directly impacts on workspace features, mechanical simplicity and number of actuators required for the reconfiguration.

The following requirements were considered to implement the reconfiguration of Parallix robot: it must be mechanically simple, without adding weight and/or actuators into the kinematic chains, be able to change the entire workspace without modifying the lengths of links, increasing the versatility without to couple or uncouple links into mechanism and minimizing the structural space occupied by the robot. In Figure 2, a conceptual design and geometrical parameters of an asymmetric reconfigurable robot that fulfills the above conditions is shown.

The reconfigurable manipulator of Figure 2 comprises of a reconfigurable fixed platform with a fixed element and three mobile elements in form of square bar moved by motors. Such motors move the square bars with simultaneous or independent movements between each other. 


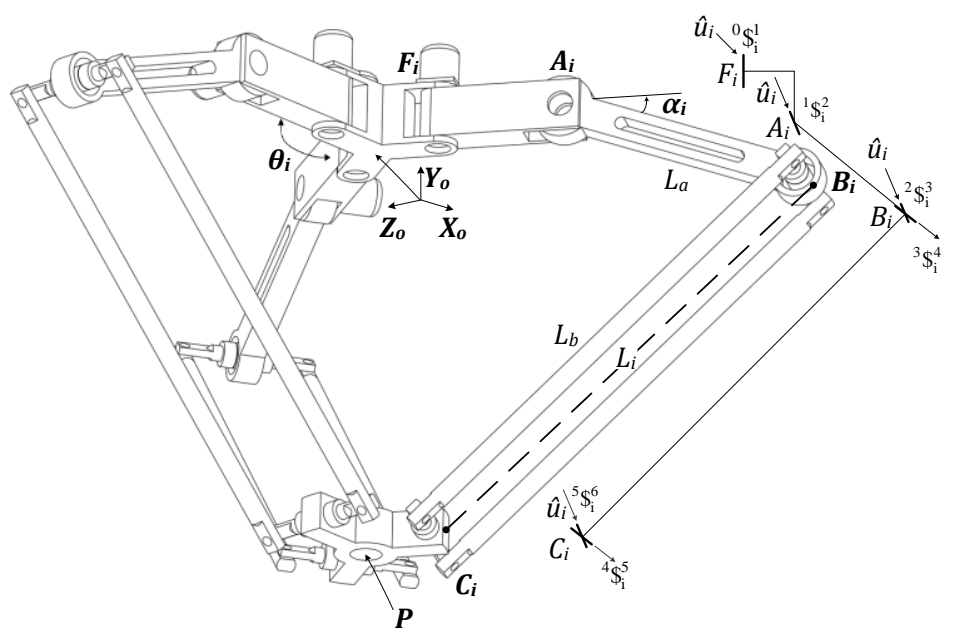

Figure 2. Reconfiguration strategy

Each square bar modifies the angle between each of the kinematic chains, thus generating asymmetric configurations of the robot. It should be note that this reconfiguration is highly versatile, since the mechanism can be reconfigured also with each of the square bar with decoupled movements from the rest of the mechanism and be able to return to the original configuration and mechanical characteristics of the Parallix LKF-2040 robot.

\section{Kinematic analysis}

\subsection{Inverse displacement analysis}

Inverse displacement analysis consists of finding the generalized coordinates $\alpha_{i}$ given the coordinates of the point $P=(P x, P y, P z)$, see Fig. 2. Now, let $X_{o} Y_{o} Z_{o}$ be the fixed reference system in the center of the fixed platform of the manipulator. The coordinates of the points $F_{i}=\left(F x_{i}, F y_{i}, F z_{i}\right), A_{i}=\left(A x_{i}, A y_{i}, A z_{i}\right)$ and $C_{i}=$ $\left(C x_{i}, C y_{i}, C z_{i}\right)$ are calculated by projections as follows

$$
\begin{aligned}
& F_{i}=\left[\begin{array}{lll}
R_{f} \cos \left(\theta_{i}\right) & 0 & R_{f} \sin \left(\theta_{i}\right)
\end{array}\right] \\
& A_{i}= {\left[\begin{array}{lll}
R_{r} \cos \left(\theta_{i}\right)+F x_{i} & 0 & R_{r} \sin \left(\theta_{i}\right)+F z_{i}
\end{array}\right] } \\
& C_{i}=\left[\begin{array}{lll}
P x+r_{m} \cos \left(\theta_{i}\right) & P y & P z+r_{m} \sin \left(\theta_{i}\right)
\end{array}\right]
\end{aligned}
$$

where $i=1,2,3 ; R_{f}$ is the distance from the fixed reference system to each point $F_{i} ; R_{r}$ is the distance between $F_{i}$ and $A_{i}, \theta_{i}$ is the orientation angle of each kinematic chain and $r_{m}$ is the distance from the point $P$ to each vertex of the equilateral triangle $C_{1} C_{2} C_{3}$. Evidently, the angles $\alpha_{i}$ may be computed upon the points $B_{i}=$ $\left(B x_{i}, B y_{i}, B z_{i}\right)$ as follows

$$
\alpha_{i}=\arcsin \left(\frac{B y_{i}-A y_{i}}{L a}\right)
$$

In order to compute $B y_{i}$ consider the following closure equations

$$
\left.\begin{array}{c}
\left(B_{i}-A_{i}\right) \bullet \widehat{u}_{i}=0 \\
\left(B_{i}-A_{i}\right) \bullet\left(B_{i}-A_{i}\right)=L a^{2} \\
\left(C_{i}-B_{i}\right) \bullet\left(C_{i}-B_{i}\right)=L b^{2}
\end{array}\right\}
$$


A. L Balmaceda-Santamaría and A. E. Chávez-Toruño.

It is worth noting that the reconfiguration does not complicate the inverse displacement analysis, but it retains the calculation simplicity of a Delta-type robot using this method.

\subsection{Manipulator workspace}

With the aim to show the shape of the workspace and to obtain its approximate volume generated by the reconfiguration, the inverse displacement analysis is applied. By using (1) and (2) and defining configurations for $0^{\circ} \leq \theta_{1} \leq 115^{\circ}, 120^{\circ} \leq \theta_{2} \leq 235^{\circ}$ and $240^{\circ} \leq \theta_{3} \leq 355^{\circ}$, the volume is obtained by sweeping the 3 dimensional space $S O(3)$ and discarding any non-real solution (Figure 3 ). It should be noted that when $\theta_{i}=120^{\circ}$ between each other, the workspace of the original configuration of the robot Parallix LKF-2040 is produced (Figure 3a).

The dimensions using SI units considered to compute the manipulator workspace are: $L a=0.2, L b=0.4, r_{m}$ $=0.05, R_{f}=0.05$ and $R_{r}=0.1118$.

In Figure $3 \mathrm{a}$ the volume obtained is $0.0698 \mathrm{~m}^{3}$. While in Figure $3 \mathrm{~b}$ the volume is $0.2223 \mathrm{~m}^{3}$, which corresponds to the union of workspaces for different configurations of the robot. Both the figures and the volumes are calculated by software Geomagic ${ }^{\circledR}$.

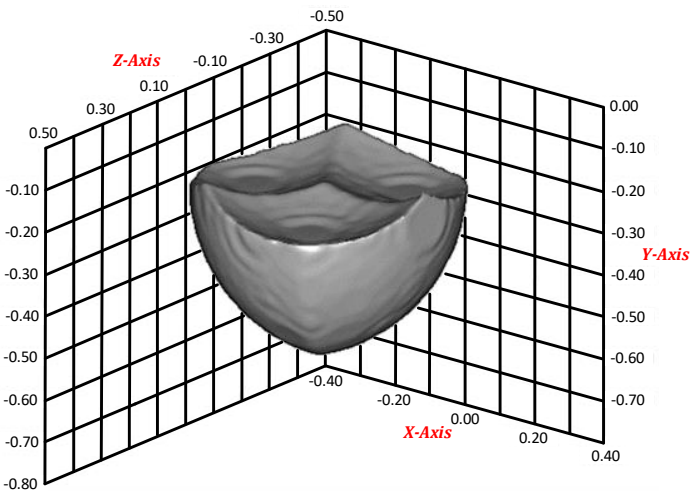

(a)

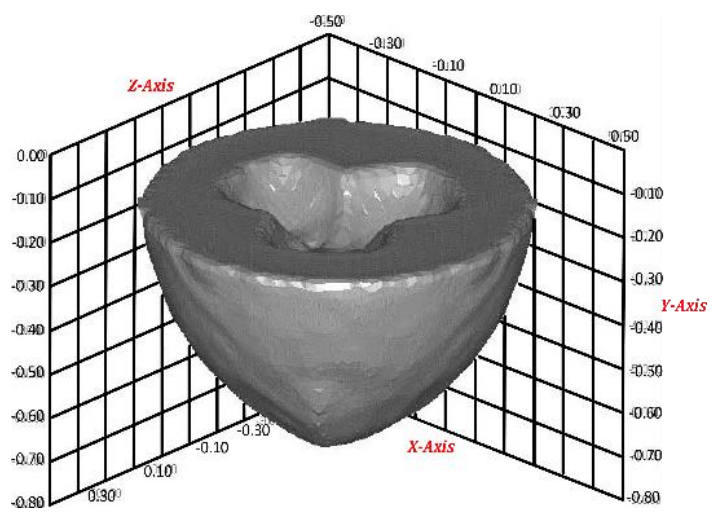

(b)

Figure 3. Reconfigurable robot workspace

In Figure $3 b$, it is evident that the workspace was limited to $-0.1 \mathrm{~m}$ in $\mathrm{Y}$-axis for discarding any collisions between the links. It is worth noting that by the reconfiguration effects the robot workspace has significantly increased, which is an additional advantage of the asymmetric reconfiguration.

\subsection{Velocity analysis}

Velocity analysis involves determining the velocity state of the moving platform with respect to the fixed platform (Balmaceda-Santamaría and García-Murillo, 2020), when the joint rates $\dot{\theta}_{4 i}=\dot{q}_{4 i}$ and $\dot{\alpha}_{i}=\dot{q}_{i}$ are given.

Let $\mathbf{V}=[0, \mathbf{v}]^{T}$ be the velocity state of the moving platform with respect to the fixed platform, where $\mathbf{v}$ is the velocity vector of any point on the moving platform. The velocity state $\mathbf{V}$ may be defined in screw form through the limbs of the robot as follows

$$
\mathbf{V}=\dot{q}_{4 i}{ }^{0} \$_{i}^{1}+\dot{q}_{i}{ }^{1} \$_{i}^{2}+{ }_{2} \omega_{3}^{i 2} \$_{i}^{3}+{ }_{3} \omega_{4}^{i}{ }^{3} \$_{i}^{4}+{ }_{4} \omega_{5}^{i}{ }^{4} \$_{i}^{5}+{ }_{5} \omega_{6}^{i}{ }^{5} \$_{i}^{6}
$$


A. L Balmaceda-Santamaría and A. E. Chávez-Toruño.

therein $i$ denote the $i$-th kinematic chain. In order to obtain an input-output velocity equation of the reconfigurable manipulator, consider that $L_{i}=\left[l_{i} l y_{i} l z_{i} L x_{i} L y_{i} L z_{i}\right]^{T}$ is a line in Plücker coordinates, which passes through the points $B_{i}$ and $C_{i}$ (Figure 2), reciprocal to all the screws in the same limb, excepting the screws associated to the active joints. Therefore, the systematic application of the Klein form, denoted as $\{* ; *\}$, of the line $L_{i}$ to both sides of (3) and reducing terms, yields the input-output velocity equation as

$$
\mathbf{v}=\mathbf{A}^{-1}\left(\mathbf{B}^{\prime} \dot{\mathbf{q}}_{4}+\mathbf{B} \dot{\mathbf{q}}\right)
$$

therein $\mathbf{A}=\left[\begin{array}{lll}l x_{i} & l y_{i} & l z_{i}\end{array}\right]^{T}$ is the Jacobian matrix, while $\dot{\mathbf{q}}_{4}=\left[\begin{array}{lll}\dot{q}_{41} & \dot{q}_{42} & \dot{q}_{43}\end{array}\right]^{T}$ and $\dot{\mathbf{q}}=\left[\begin{array}{lll}\dot{q}_{1} & \dot{q}_{2} & \dot{q}_{3}\end{array}\right]^{T}$ are the firstorder generalized coordinates of the robot. The passive Jacobian are $\mathbf{B}^{\prime}=\operatorname{diag}\left[\left\{{ }^{0} \$_{1}^{1} ; L_{1}\right\} \quad\left\{{ }^{0} \$_{2}^{1} ; L_{2}\right\} \quad\left\{{ }^{0} \$_{3}^{1} ; L_{3}\right\}\right]$ and $\mathbf{B}=\operatorname{diag}\left[\left\{{ }^{1} \$_{1}^{2} ; L_{1}\right\} \quad\left\{{ }^{1} \$_{2}^{2} ; L_{2}\right\} \quad\left\{{ }^{1}{ }_{3}^{2} ; L_{3}\right\}\right]$.

\section{Numerical example}

In this section, a numerical example is provided in order to show that by redundancy it is possible to obtain customized solutions of the kinematic performance. For this aim, the compute of the condition number is used as a performance index throughout defined trajectories (see Figure 4).

Two Cartesian trajectories of the center $P$ of the moving platform are generated by solving the direct displacement analysis (Gallardo-Alvarado, Balmaceda-Santamaría and Castillo-Castaneda, 2014), considering an interval for time $t$ of $0 \leq t \leq 2 \pi$. The link lengths used are the same as in the previous Section and the direction of rotation axes of the active joints are defined by $\hat{u}_{1}=-\hat{k}, \hat{u}_{2}=-0.866 \hat{\imath}+0.5 \hat{k}$ and $\hat{u}_{3}$ $=0.866 \hat{\imath}+0.5 \hat{k}$.

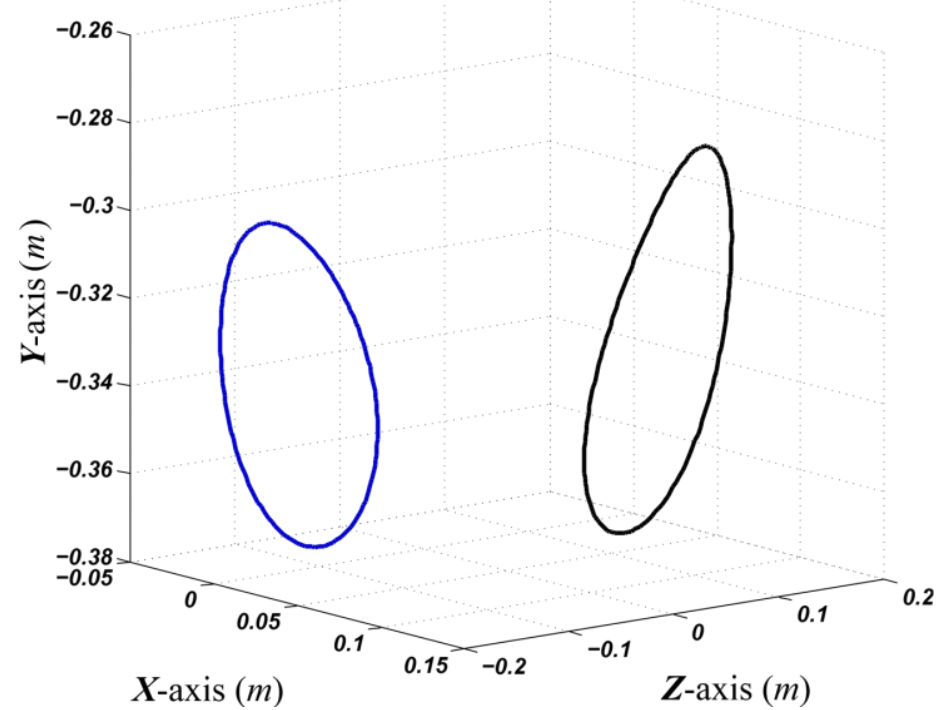

Figure 4. Trajectories of the center $P$ of the manipulator.

The trajectories in Figure 4 are performed in different planes and considering the three degrees of freedom of the mechanism. 


\subsection{Velocity analysis}

In (Merlet, 2007) is defined that the condition number describes the accuracy/dexterity and the closeness to a singular configuration of a parallel manipulator. For this reason, many authors (as some of those aforementioned) have used the condition number as a performance index of a Delta-type robot. In our case, the results of this performance index are improved by using the redundancy generated by the proposed reconfiguration.

To have a better appreciation of the condition number, the inverse condition number $k^{-1}$ is used, since it has a range between $0 \leq k^{-1} \leq 1$. The inverse condition number is defined as

$$
k^{-1}=\frac{1}{\left\|\mathbf{J}^{-1}\right\|\|\mathbf{J}\|}
$$

where $\mathbf{J}$ is the manipulator Jacobian matrix, obtained from (4) as follows

$$
\mathbf{v}=\left(\mathbf{A}^{-1} \mathbf{B}\right) \dot{\mathbf{q}}=\mathbf{J} \dot{\mathbf{q}}
$$

since the matrix $\mathbf{J}$ of the well-known architecture of the Delta-type robot is used, in order to demonstrate that it is possible to enhance its kinematic performance by the redundancy of the asymmetric reconfigurable mechanism.

It should be mentioned that when $k^{-1}=0$ Jacobian matrix is not invertible, hence the manipulator cannot generate velocities in some directions which is called, being at a singular configuration (Tsai, 1999). While that $k^{-1}=1$, then the manipulator is capable of generating velocities in any direction, in other words being at an isotropic pose (Merlet, 2007).

Note that the first term of (4) depends on $\dot{\theta}_{i}$ and $k\left(\dot{\theta}_{i}\right)$, since the best solution of $k^{-1}$ is computationally obtained by sweeping all possible combinations of $\theta_{i}$ in the same range defined in subsection 3.2 and seeking locally for each one of the trajectory points.

The best results of $k^{-1}$ are obtained for the 181 points of each trajectory by using the redundancy of the reconfigurable robot and they are compared with the result of $k^{-1}$ obtained with the original configuration of the Delta-type robot (see Figure 5).

In Figure 5a is evident that with the asymmetric reconfigurable manipulator a remarkable improved in performance index can be obtained. Meanwhile with the original Delta-type Parallix robot a performance index lower than 0.2 is obtained.

It should be note that in Figure $5 \mathrm{~b}$ a similar behavior is obtained for the asymmetric reconfigurable mechanism, since as can be observed the performance index is upper than 0.7 at the begin, in middle and at the end of the black trajectory of Figure 4. 
A. L Balmaceda-Santamaría and A. E. Chávez-Toruño.

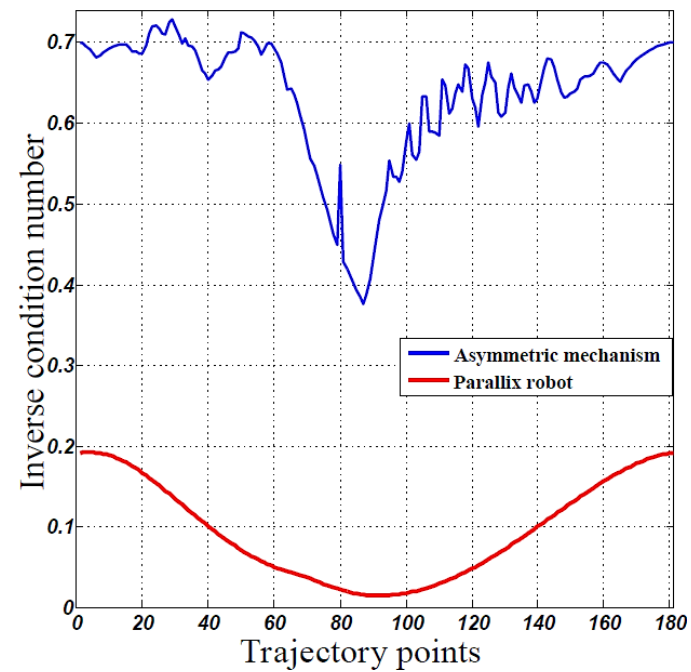

(a)

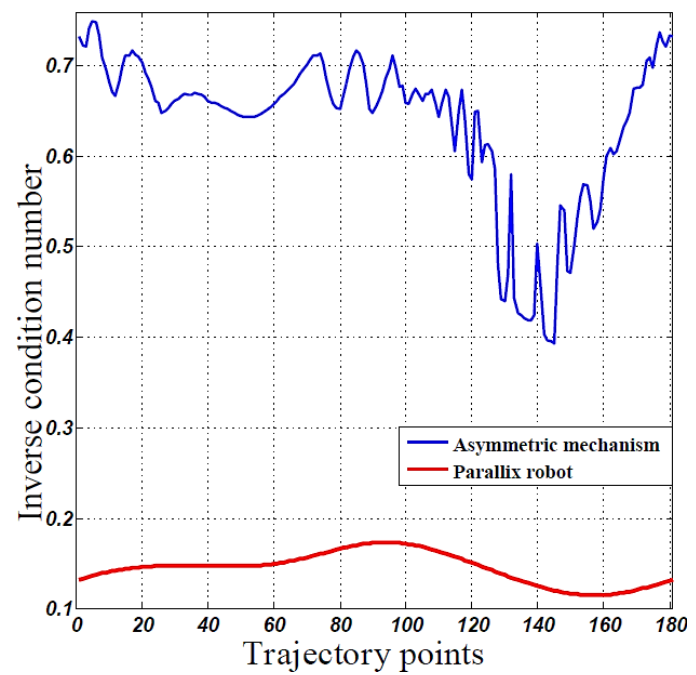

(b)

Figure 5. Value of $k^{-1}$ for each trajectory of Figure 4. Blue circle (a), black circle (b).

In each point of the prescribed trajectories, the reconfigurable mechanism obtains values of $k^{-1}$ nearest to 1 , which could not be achieved only with the Delta-type robot. Therefore, since the results of the condition number are improved in each trajectory thanks to the asymmetric reconfiguration, all the other performance indices that depend on the manipulator Jacobian matrix, e.g.: Manipulability index, Minimum singular value, Global conditioning index, Global manipulability index and even the stiffness, are also improved.

\section{CONCLUSIONS}

The reconfigurability in parallel robots is still a challenge nowadays, since most of such reconfiguration strategies have a high level of mechanical complexity in their structures and hence they are very expensive to be made. However, a very limited number of authors have proposed reconfigurable Delta robots.

In this paper, an asymmetric reconfiguration of a Delta-type parallel robot is proposed. This reconfiguration proposed is highly versatile, very simple and it can be able to return to the original configuration and mechanical characteristics of a Delta-type robot.

The reconfiguration proposal, consists of a fixed platform comprised by a fixed element and mobile elements, which can modify the angle between each of the kinematic chains, thus generating asymmetric configurations of the mechanism.

It is very important to note that, by the reconfiguration proposal significant advantages can be obtained with respect to a Delta-type parallel robot.

The velocity analysis is reported by resorting to reciprocal-screw theory. Moreover, customized solutions of the robot kinematic performance can be obtained by using the redundancy produced by the reconfiguration. Additional advantages such as: increased workspace, can be obtained.

A numerical example of the manipulator performance is provided. The computation of the best condition number is obtained, according to prescribed trajectories to different heights of the robot workspace. The results show that by using the redundancy, the condition number is enhanced in each trajectory. Accordingly, all the performance indices that depend on the Jacobian matrix are also improved. 
A. L Balmaceda-Santamaría and A. E. Chávez-Toruño.

\section{REFERENCIAS}

Balmaceda-Santamaría A. L., García-Murillo M. A. (2020) Kinematics of a Planar Parallel Robot via Screw Theory: Details not Mentioned. In: Kuo CH., Lin PC., Essomba T., Chen GC. (eds) Robotics and Mechatronics. ISRM 2019. Mechanisms and Machine Science, Springer, Cham, vol 78, 91 - 102.

Balmaceda-Santamaría, A. L. and Chavez-Toruño A. E (2019). Redundant reconfigurable Delta-type parallel robot, 2019 IEEE 4th International Conference on Advanced Robotics and Mechatronics (ICARM), 292 - 297.

Bi, Z. M., Kang, B. (2010). Enhancement of adaptability of parallel kinematic machines with an adjustable platform. Journal of Manufacturing Science and Engineering, Vol. 132 (6).

Borràs, J., Thomas, F., Ottaviano, E., Ceccarelli, M. (2009). A reconfigurable 5-DoF 5-SPU parallel platform, ASME/IFToMM International Conference on Reconfigurable Mechanisms and Robots, 617 - 662.

Carbonari, L., et al. (2014). A new class of reconfigurable parallel kinematic machines. Mechanism and Machine Theory 79, $173-183$.

Cha, S-H., Lasky, T. A., Velinsky, S. A. (2007). Singularity avoidance for the 3-RRR mechanism using kinematic redundancy. IEEE International Conference on Robotics and Automation, 10 - 14.

Chen, Chun-Ta. (2012). Reconfiguration of a parallel kinematic manipulator for the maximum dynamic load-carrying capacity. Mechanism and Machine Theory 54, 62 - 75.

du Plessis, L.J., Snymar, J.A. (2006). An optimally reconfigurable planar Gough-Stewart machining platform. Mechanism and Machine Theory 41, 334 - 357.

Finistuari, A.D., Xi, F.(J). (2013). Reconfiguration analysis of a fully reconfigurable parallel robot. Journal of Mechanism and Robotics, Vol 5 (4). doi: 10.1115/1.4024734.

Gallardo-Alvarado, J., Balmaceda-Santamaría, A.L., Castillo-Castaneda, E. (2014). An application of screw theory to the kinematic analysis of a Delta-type robot. Journal of Mechanical Science and Technology, Vol. 28 (9), 3785 - 3792.

Gogu, $\mathrm{G}$ (2007). Isogliden-TaRb: a family of up to five axes reconfigurable and maximally regular parallel kinematic machines. Journal Manufacturing Systems, 36(5), 419 - 426.

Gonzalez-Hernandez, A., Castillo-Castaneda, E. (2013). Stiffness estimation of a parallel manipulator using image analysis and camera calibration techniques. Robotica, Vol. 31, 657 - 667.

Jiang, Y., Li, T., Wang, L. (2015a). The dynamic modeling, redundant-force optimization, and dynamic performance analyses of a parallel kinematic machine with actuation redundancy. Robotica, Vol. 33, 241 263.

Jiang, Y., Li, T., Wang, L. (2015b). Dynamic modeling and redundant force optimization of a 2-DOF parallel kinematic machine with kinematic redundancy, Robotics and Computer-Integrated Manufacturing, Vol. 32, 1 - 10 .

Nexo Revista Científica / Vol. 33, No. 01, pp. 137-147 / Junio 2020 
A. L Balmaceda-Santamaría and A. E. Chávez-Toruño.

Kock, S., Schumacher, W. (1998). A parallel x-y manipulator with actuation redundancy for high-speed and active-stiffness applications. Robotics and Automation, Proceedings 1998 IEEE International Conference on Vol. 3, 2295 - 2300.

Kotlarski, J., Heimann, B., Ortmaier, T (2012). Influence of kinematic redundancy on the singularity-free workspace of parallel kinematics machines. Frontiers of Mechanical Engineering, Vol. 7 (2), 120 - 134.

Kotlarski, J., Heimann, B., Ortmaier, T. (2010). Improving the pose accuracy of planar robot using mechanism of variable geometry. Advances in Robot Manipulators, InTech, 381 - 400.

Krefft, M., et al. (2006). Reconfigurable parallel robots: Combining high flexibility and short cycle times. Journal of Production Engineering, XIII (1), 109 - 112.

Kumar Satheesh, G., Nagarajan, T., Srinivasa, Y.G. (2009). Characterization of reconfigurable Stewart platform for contour generation. Robotics and Computer-Integrated Manufacturing. (25), 721 - 731.

Li, C., Zhang, X. G., Zhao, Y. S. (2014). Redundant actuation investigation of a 5-DOF parallel robot. Applied Mechanics and Materials, Vol. 526, 164 - 170.

Merlet, J. P. (2007). Jacobian, manipulability, condition number and accuracy of parallel robots. Robotics Research. Springer Tracts in Advanced robotics, Vol. 8, 175 - 184.

Moosavian, A., Xi, F. (J). (2014). Design and analysis of reconfigurable parallel robots with enhanced stiffness. Mechanism and Machine Theory 77, 92 - 110.

Plitea, N., et al. (2013). Structural design and kinematics of a new parallel reconfigurable robot. Robotics and Computer-Integrated Manufacturing 29, 219 - 235.

Simaan, N., Shoham, M. (2003). Stiffiness synthesis of a variable geometry six-degrees-of-freedom double planar parallel robot. The International Journal of Robotics Research, Vol. 22, No. 9, 757 - 775.

Simaan, N., Shoham, M. (2003). Stiffness synthesis of a variable geometry six-degrees-of- freedom double planar parallel robot. The International Journal of Robotics Research, 22 (9), 757 - 775.

Tsai, L.W., (1999). Robot analysis: The mechanics of serial and parallel manipulators. United States of America, Wiley-interscience publication.

Wang, D. Fan, R., Chen, W. (2014). Performance enhancement of a three-degree-of-freedom parallel tool head via actuation redundancy. Mechanism and Machine Theory 71, 142 - 162.

Wu, J. Wang, J. Wang, L (2008). Optimal kinematic design and application of a redundantly actuated 3DOF planar parallel manipulator. Journal of Mechanical Design, Vol. 130 (5) doi: 10.1115/1.2890118.

Xu, B., Li, T. X., Liu, J. W. (2010). Workspace Analysis of the 4RRR Planar Parallel Manipulator with Actuation Redundancy. Tsinghu Science and Technology, Vol. 15 (5), 509 - 516.

Ye, W., et al. (2014). A new family of reconfigurable parallel mechanism with diamond kinematotropic chain. Mechanism and Machine Theory, Vol. 74, 1 - 9.

Yue, S., Tso, S. K., Xu, W. L. (2001). Maximum-dynamic-payload trajectory for flexible robot manipulators with kinematic redundancy. Mechanism and Machine Theory Vol. 36, 785 - 800.

Nexo Revista Científica / Vol. 33, No. 01, pp. 137-147 / Junio 2020 
A. L Balmaceda-Santamaría and A. E. Chávez-Toruño.

Zhang, D., Shi, Q. (2012). Novel design and analysis of a reconfigurable parallel manipulator using variable geometry approach. Practical Applications of Intelligent Systems. Advances in Intelligent and Soft Computing. Vol. 124, 447 - 457.

Zhiming, J., Phillip, S (1998). Design of a reconfigurable platform manipulator. Journal of Robotic Systems 15(6), $341-346$.

\section{SEMBLANZA DE LOS AUTORES}

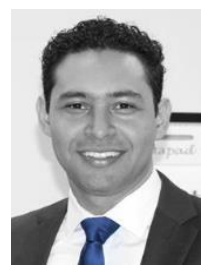

Albert L. Balmaceda-Santamaría: Graduated in Industrial Engineering at National University of Engineering, Nicaragua in 2009. He received the Master and $\mathrm{Ph}$. D. degrees in advanced technology at IPN - CICATA, Queretaro, Mexico in 2011 and 2016, respectively. He did research stays at the Celaya Institute of Technology, Guanajuato, México in Kinematics and Dynamics of parallel robots by means Screw Theory. Currently, He is a researcher professor at National University of Engineering, Nicaragua. Dr. Balmaceda-Santamaría does research in Industrial Engineering, Manufacturing Engineering, Screw Theory, Industrial Parallel Robots, Mechanical Engineering and Design. In addition, he is the inventor of two reconfigurable parallel robot patents.

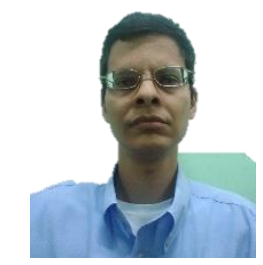

Ariel E. Chávez-Toruño: Graduated in Systems Engineering at National University of Engineering, Nicaragua in 2001. He received the Master degree in Business Computing at National University of Engineering, Nicaragua in 2013. Nowadays, He is an outstanding professor of the Faculty of Science and Systems in several application fields as: Programming, Software Engineering, Manufacturing Systems, Artificial Intelligence and many others. Actually, He works on Artificial Intelligence, Technology and Innovation.

Nexo Revista Científica / Vol. 33, No. 01, pp. 137-147 / Junio 2020 